\title{
Orientación sobre lactancia materna en un hospital para enfermedades diarreicas ${ }^{1}$
}

\author{
R. Haider, ${ }^{2}$ A. Islam, ${ }^{2}$ J. Hamadani, ${ }^{2}$ N. J. Amin, ${ }^{3}$ I. Kabir, ${ }^{2}$ \\ M. A. Malek, ${ }^{3}$ D. Mahalanabis ${ }^{2}$ y D. Habte ${ }^{2}$
}

RESUMEN Se adiestró a consejeras en materia de lactancia materna para que dieran asesoramiento a las madres de lactantes parcialmente amamantados y hospitalizados por diarrea, con el fin de lograr que las madres iniciaran la lactancia materna exclusiva durante su permanencia en el hospital. Se asignó de forma aleatoria a lactantes $(\mathrm{n}=250)$ de 12 meses de edad o menos a un grupo de intervención y a un grupo testigo. Las madres del grupo de intervención fueron asesoradas individualmente por las consejeras, mientras que las madres del grupo testigo recibieron solo la educación sanitaria impartida en grupo rutinariamente. Durante el seguimiento que hicieron las consejeras en el hogar una semana más tarde, solo las madres del grupo de intervención fueron asesoradas. A las 2 semanas de su egreso, todas las madres fueron evaluadas en términos de sus prácticas de amamantamiento en el hogar.

En cuanto a los 125 pares de madres e hijos que había en cada grupo, 60\% de las madres del grupo de intervención alimentaban a sus hijos al pecho exclusivamente en el momento del egreso, en comparación con solo $6 \%$ de las madres del grupo testigo $(\mathrm{P}<0,001)$; 2 semanas más tarde, estos porcentajes subieron a 75 y 8 en el grupo de intervención y en el grupo testigo, respectivamente $(\mathrm{P}<0,001)$. Sin embargo, $49 \%$ de las madres del grupo testigo volvieron a alimentar a sus hijos con biberón, en comparación con $12 \%$ de las madres del grupo de intervención ( $\mathrm{P}<0,001)$. Por consiguiente, la orientación individual tuvo un efecto favorable en las madres, ya que hizo que iniciaran la lactancia materna exclusiva durante la hospitalización y que la siguieran practicando en el hogar.

Los centros de salud maternoinfantil deben incluir la orientación sobre lactancia materna como parte integral de sus programas para mejorar las prácticas de alimentación de los lactantes.

A pesar de la alta prevalencia de la lactancia materna, solo una pequeña

\footnotetext{
Perteneciente al Centro Internacional para la Investigación de las Enfermedades Diarreicas y al Instituto de Nutrición y Ciencias de la Alimentación, Universidad de Dacca, Dacca, Bangladesh. Se publicó en inglés en el Bulletin of the World Health Organization, Vol. 74, No. 2, 1996, con el título "Breast-feeding counselling in a diarrhoeal disease hospital". (c) Organización Mundial de la Salud, 1996.

2 Centro Internacional para la Investigación de las Enfermedades Diarreicas, Dacca, Bangladesh. Las solicitudes de separatas deben dirigirse a R. Haider a la siguiente dirección postal: Clinical Sciences Division, ICDDR, B, GPO Box 128, Dhaka 1000, Bangladesh.

3 Instituto de Nutrición y Ciencias de la Alimentación, Universidad de Dacca, Dacca, Bangladesh.
}

proporción de los lactantes menores de 4 meses en países en desarrollo son amamantados al pecho exclusivamente (1). La morbilidad (2-4) y mortalidad (5) por enfermedades diarreicas se incrementan cuando se introducen papillas $\mathrm{u}$ otros tipos de leche. Asimismo, los lactantes amamantados que contraen shigelosis $y$ cólera tienen un menor riesgo de enfermar de gravedad $(6,7)$. Los estudios efectuados en países donde son bajas las tasas de mortalidad en lactantes revelan que los niños alimentados con leche artificial requieren trata- miento en el hospital con una frecuencia que es hasta cinco veces mayor que la observada en niños amamantados total o parcialmente (8). La lactancia materna exclusiva proporciona, sin embargo, la máxima protección $(8,9)$. Lamentablemente en Bangladesh, donde la mayoría de las mujeres amamantan a sus hijos, casi no se practica la lactancia materna exclusiva (10).

La OMS ha estimado que el fomento de la lactancia materna podría llevar a una reducción de $25 \%$ de la mortalidad por enfermedades diarreicas en los primeros 6 meses de vida $(11,12)$; también 
se ha calculado la eficacia de esa intervención en relación con su costo. ${ }^{4}$ Las estrategias para el fomento de la lactancia materna se han concentrado en madres cuyos hijos nacen en el hospital o en un servicio de salud (13), pero en muchos países en desarrollo (como por ejemplo, en Bangladesh) pocos partos se producen en esas instituciones. Por otra parte, a los lactantes se les lleva a los servicios de salud cuando están enfermos y la oportunidad de ejercer alguna influencia en las madres durante estas visitas no se debe perder. En el hospital del Centro Internacional para la Investigación de las Enfermedades Diarreicas, situado en Dacca, Bangladesh, la proporción de lactantes pequeños hospitalizados por enfermedades diarreicas ha aumentado progresivamente con el pasar de los años. De los 3058 lactantes menores de 3 meses que fueron llevados al hospital durante el período de 1993 a 1994, 63\% habían recibido lactancia materna parcial y $28 \%$ no habían sido amamantados (datos inéditos).

El presente estudio se realizó, por lo tanto, con el fin de aconsejar a las madres que llevaron al hospital a sus lactantes pequeños para el tratamiento de la diarrea que iniciaran la lactancia materna exclusiva durante la hospitalización y que continuaran la práctica en el hogar hasta que el bebé cumpliera 6 meses de edad.

\section{MATERIALES Y MÉTODOS}

En este estudio se han usado las siguientes definiciones $(14)^{5}$ :

- Lactancia materna exclusiva: se da a los lactantes únicamente leche materna (ningún otro alimento líquido o sólido);

\footnotetext{
4 Phillips MA, et al. Options for diarrhoea control: the cause and effectiveness of selective interventions for the prevention of diarrhoea. London: School of Hygiene and Tropical Medicine; 1987. (Documento inédito).

5 World Health Organization. Indicators for assessing breast-feeding practices: report of an informal meeting, 11-12 June 1991, Geneva, Switzerland. Geneva: WHO; 1991. (Documento inédito CDD/ SER/91.14).
}

- Lactancia predominantemente materna: se da leche materna más sales de rehidratación oral (SRO), agua, o ambas cosas; $y$

- lactancia materna parcial: se da leche materna más papillas u otras leches.

\section{Capacitación del personal que hizo el estudio}

Las consejeras que dieron asesoramiento sobre la lactancia materna y las médicas que participaron en la investigación (que fueron mujeres exclusivamente) recibieron un adiestramiento que duró 3 semanas. Con tal fin se ofreció el curso sobre asesoramiento en materia de lactancia materna para agentes de salud. ${ }^{6}$ A estos miembros del personal de salud también se les dio instrucción, seguida de oportunidades de práctica, sobre el tratamiento de las enfermedades diarreicas y sobre las mediciones antropométricas necesarias para hacer el estudio. Finalmente, se reclutó a algunos pacientes para hacer una prueba piloto y adquirir familiaridad con los procedimientos y cuestionarios usados en la recolección de datos.

\section{Diseño del estudio}

El estudio fue un ensayo aleatorio controlado que se inició en el hospital. Su evaluación, que se efectuó en el momento en que se dio de alta a los pacientes y más tarde (en el hogar), estuvo a cargo de médicas que no participaron en el asesoramiento. Los pares de madres e hijos fueron agrupados de la siguiente manera:

- Grupo de intervención: madres de lactantes que recibieron asesoramiento sobre lactancia materna y apoyo en la misma durante la

\footnotetext{
6 World Health Organization, United Nations Children's Fund. Breast-feeding counselling: a training course. Geneva: WHO; 1993. (Documento inédito WHO/CDD/93.3-6 y UNICEF/NUT/ 93.1-4).
}

hospitalización, con refuerzo en el hogar una semana después del egreso; $y$

- Grupo testigo: madres de lactantes que recibieron los mensajes de educación sanitaria impartidos de forma rutinaria, incluido el asesoramiento habitual sobre lactancia materna, solamente durante la hospitalización.

Ninguno de los lactantes incluidos en el estudio había dejado de mamar $\mathrm{y}$, por lo tanto, no fue necesario reiniciar la lactancia. La frecuencia del consumo de leche materna y de otros alimentos durante el día y la noche, así como la cantidad ingerida, fueron similares en ambos grupos.

\section{Criterios de inclusión y de exclusión}

Con objeto de facilitar el seguimiento, se incluyó en el estudio a los lactantes de 12 semanas de edad o menos que habían tenido diarrea un máximo de 5 días, que tenían un peso para la edad de $60 \%$ o más del peso promedio determinado por el Centro Nacional de Estadísticas Sanitarias de los Estados Unidos de América (NCHS), y que vivían en Dacca a una distancia razonable (no más de $15 \mathrm{~km}$ ). Se excluyó del estudio a los lactantes que no requerían hospitalización (estos fueron enviados a su casa de la consulta externa con SRO), que tenían infección grave, o cuyas madres no podían o no estaban dispuestas a quedarse en el hospital. También se excluyó a los lactantes que permanecieron en el hospital menos de 8 horas después de la selección. Estas exclusiones se hicieron necesarias a causa de las limitaciones de tiempo para la orientación.

\section{La selección aleatoria}

Se usaron bloques permutados aleatorios de longitud variable para seleccionar al azar el grupo de intervención y el grupo testigo. Se colocaron en sobres cerrados los números seriados 
correspondientes a la lista aleatoria. Después de haberse obtenido el consentimiento de los padres, debidamente informados de los propósitos del estudio como lo exige el Comité de Revisión Ética, cada pareja de madre e hijo fue asignada al grupo de intervención o al grupo testigo según la secuencia numérica indicada en los sobres.

\section{El tratamiento estándar de los casos}

Se elaboró una historia clínica de base para registrar toda la información que fuera pertinente sobre la alimentación del niño en ese momento y en el pasado y se examinó al paciente. Se rehidrató a los lactantes y la hidratación se mantuvo con líquidos intravenosos o SRO. Se siguió amamantando a los lactantes al pecho durante toda su hospitalización y los que debían recibir un alimento formulado para lactantes fueron alimentados con taza y cuchara, conforme a la política del hospital. La investigación y el tratamiento de las infecciones se hicieron en conformidad con las prácticas habituales del hospital del Centro Internacional para la Investigación de las Enfermedades Diarreicas.

Se programó someter a las madres incluidas en el grupo de intervención a un mínimo de tres sesiones de orientación, que serían impartidas por las consejeras en lactancia el día 1 y el día del egreso, y por el investigador principal el día 2. La primera sesión fue breve (tardó de 5 a 7 minutos) porque se consideró que las madres estaban preocupadas y ansiosas por la enfermedad de sus hijos $\mathrm{y}$, por lo tanto, prestarían menos atención. Las siguientes dos sesiones fueron más largas y tuvieron una duración aproximada de 30 a 40 minutos. Las consejeras en lactancia primero trataron de determinar las razones por las cuales se había iniciado tempranamente la administración de alimentos complementarios, y luego proporcionaron la información pertinente sobre la lactancia materna (es decir, que contribuye a la recuperación de la diarrea, que cuanto más amamanta más leche tiene la madre y que la leche materna sola basta para el crecimiento del bebé durante los primeros 5 meses de vida) y sobre la importancia de una dieta materna adecuada y del apoyo familiar necesario para que pueda amamantar. En cada sesión se cubrieron solo tres o cuatro aspectos importantes de la lactancia materna. Las consejeras también ayudaron a las madres, cuando era necesario, a colocar al bebé en la posición adecuada para que tomara el pezón. Las madres del grupo testigo concurrieron diariamente a las sesiones de educación sanitaria de carácter rutinario, en las que se incluyó orientación sobre la práctica durante un período de 5 meses de la lactancia materna exclusiva, seguida de la adición de alimentos complementarios preparados en el hogar.

\section{El seguimiento}

Una semana después del egreso, una consejera en lactancia materna siguió en el hogar a los pares de madres e hijos que integraban el grupo de intervención y el grupo testigo. A las madres del primero se les hicieron preguntas sobre la alimentación de los lactantes (por recordación de las últimas 24 horas), se les dio asesoramiento sobre cualquier problema de alimentación y salud, y se las instó a seguir amamantando. Las madres del grupo testigo también fueron visitadas por las mismas consejeras, que les hicieron preguntas similares pero sin darles asesoramiento en materia de lactancia materna. Las consejeras pasaron de 2 a 4 horas en las casas de las entrevistadas, observando la alimentación de los lactantes y averiguar las opiniones de los miembros de la familia sobre el amamantamiento. Durante estas visitas también se tomó nota de los enseres domésticos, del tipo de letrina y del agua usada para beber y lavar. Al cabo de 2 semanas de seguimiento domiciliario, una médica que participó en el estudio efectuó una evaluación (de los datos recordados sobre la lactancia del niño en las últimas 24 horas, de la morbilidad durante el período de 2 sema- nas, y de las observaciones estructuradas del amamantamiento).

\section{Evaluación y monitoreo del personal del estudio}

Las consejeras en lactancia fueron monitoreadas por el investigador principal (RH), que examinó las evaluaciones del amamantamiento mediante observaciones directas y frecuentes. Se pidió a cada consejera que evaluara un episodio de amamantamiento (la posición de la madre y del niño y la adherencia de este al pezón, etc.) y que tomara mediciones antropométricas de las madres y lactantes. Posteriormente esas evaluaciones se compararon a fin de determinar el grado de variación interobservador. Se monitoreó de forma similar a las médicas que participaron en el estudio en relación con el tratamiento de la diarrea, la recopilación de datos durante el seguimiento, y la anotación de los datos.

\section{Los métodos estadísticos}

Después de evaluar los datos de base para determinar la comparabilidad de los pares de madres e hijos en los grupos de intervención y testigo, se compararon las variables resultantes (la situación del amamantamiento en el momento de dar de alta a los niños y en el hogar 2 semanas más tarde, el empleo de biberones, el peso del bebé, etc.) mediante pruebas paramétricas y no paramétricas. Se sometieron a regresión logística las variables que podrían afectar a la alimentación del lactante, incluidos su edad y sexo, la edad y escolaridad de la madre y los ingresos del padre.

\section{RESULTADOS}

Se incluyó en el estudio a un total de 250 pares de madres e hijos, 125 en cada grupo. La edad, el estado nutricional y la duración de la diarrea de los lactantes del grupo de intervención y del grupo testigo eran similares 
(cuadro 1). Veintidós (18\%) de los lactantes del grupo testigo y $25(20 \%)$ de los pertenecientes al grupo de intervención tenían deshidratación moderada o grave en el momento de la hospitalización y fueron tratados con líquidos intravenosos para su rehidratación inicial; 17 (14\%) de los lactantes de cada grupo tenían además una infección asociada de las vías respiratorias. Ambos grupos también eran similares en cuanto a la escolaridad y el estado nutricional de las madres, así como a la escolaridad, ocupación e ingresos de los padres (cuadro 2). En el grupo de intervención fueron más los lactantes primogénitos (65, en comparación con $44(P=0,007))$, mientras que en el grupo testigo fueron más los lactantes que eran el segundo hijo (45, en comparación con $30(P=0,03))$. Sin embargo, no se encontró ninguna asociación entre el orden de nacimiento y la lactancia materna exclusiva cuando se aplicaron la prueba de chi al cuadrado $\left(\chi^{2}\right)$ y la regresión logística para investigar la relación. Tampoco se detectó ninguna asociación entre el sexo del bebé y la lactancia materna exclusiva. Las viviendas e instalaciones sanitarias de las familias también fueron similares en ambos grupos. La mayoría de los lactantes (85\% en el grupo testigo y $80 \%$ en el grupo de intervención) habían recibido alimentos prelácteos después del parto (miel, agua azucarada, agua sola, etc.), seguidos de calostro. En el grupo testigo y en el de intervención la alimentación complementaria se inició aproximadamente en el mismo momento: a los $16,5 \pm 16,6$ días y a los $17,6 \pm 18,2$ días, respectivamente (cuadro 3 ).

La duración media de la hospitalización \pm una desviación estándar (DE) fue de 4,3 $\pm 1,8$ días en el grupo de intervención y de 3,0 $\pm 2,0$ días en el grupo testigo $(P<0,001)$. En general, los lactantes del grupo testigo salieron del hospital antes de que hubiera desaparecido la diarrea, que es el proceder habitual en el hospital, mientras que a los del grupo de intervención se los instó a permanecer hospitalizados hasta que hubiera desaparecido la diarrea, con el fin de asegurar suficiente oportunidad y tiempo para la modifi-

CUADRO 1. Características de los lactantes del estudio en el momento de la hospitalización

\begin{tabular}{lcc}
\hline & $\begin{array}{c}\text { No. en } \\
\text { el grupo testigo } \\
(n=125)\end{array}$ & $\begin{array}{c}\text { No. en } \\
\text { el grupo de intervención } \\
(n=125)\end{array}$ \\
\hline Edad (días): & $53,8 \pm 18,3^{\mathrm{a}}$ & $51,0 \pm 19,8^{\mathrm{a}}$ \\
$<1$ mes & $15(12)^{\mathrm{b}}$ & $24(19)$ \\
$1-2$ meses & $66(53)$ & $54(43)$ \\
$2-3$ meses & $44(35)$ & $47(38)$ \\
Mujeres & $45(36)$ & $40(32)$ \\
Varones & $80(64)$ & $85(68)$ \\
Peso para la edad & & $76,9 \pm 11,5 \mathrm{~b}^{\mathrm{a}}$ \\
(\% de la mediana del $\left.\mathrm{NCHS}^{\mathrm{c}}\right)$ & $78,6 \pm 11,6^{\mathrm{a}}$ & $90,8 \pm 10,7^{\mathrm{a}}$ \\
Peso para la talla & & \\
(\% de la mediana del $\left.\mathrm{NCHS}^{\mathrm{c}}\right)$ & $91,8 \pm 10,8^{\mathrm{a}}$ & $116(93)$ \\
Residencia: & & $2(2)$ \\
Ciudad & $114(91)$ & $7(6)$ \\
Tugurio & $4(3)$ & \\
Zona periurbana & $7(6)$ & $93(74)$ \\
Nacido en: & & $25(20)$ \\
Casa & $93(74)$ & $7(6)$ \\
Hospital & $22(18)$ & $3,5 \pm 1,7^{\mathrm{a}}$ \\
Clínica & $10(8)$ & \\
Días con diarrea & $3,6 \pm 1,6 \mathrm{a}^{\mathrm{a}}$ & \\
\hline
\end{tabular}

a Promedio \pm desviación estándar.

${ }^{b}$ Las cifras entre paréntesis son porcentajes.

${ }^{c}$ Centro Nacional de Estadísticas Sanitarias, Estados Unidos de América.

cación del comportamiento. En el momento en que egresaron del hospital, $74(60 \%)$ de las madres que habían recibido asesoramiento en materia de lactancia materna ya habían empezado a practicar la lactancia exclusiva, en comparación con solo siete (6\%) de las madres en el grupo testigo. Como muchos de los lactantes regresaron a sus hogares antes de la desaparición de la diarrea, $37(30 \%)$ de las madres del grupo que recibió asesoramiento practicaban la lactancia predominantemente materna (leche materna $+\mathrm{SRO}$ ) en el momento en que sus hijos egresaron, mientras que $24(19 \%)$ de las madres del grupo testigo practicaban esa forma de lactancia $(P<0,001)$. No se observaron diferencias entre las tasas de lactancia materna exclusiva de las madres que habían recibido dos y tres sesiones de asesoramiento durante su permanencia en el hospital.

Dos semanas más tarde en el hogar, cuando se interrumpió la administración de SRO, la frecuencia de lactancia materna exclusiva aumentó a 78 casos (75\%) en el grupo de intervención, en comparación con solo $8(8 \%)$ en el grupo testigo (figura 1). Por otra parte, $49 \%$ de las madres del grupo testigo habían vuelto a dar el biberón, en comparación con solo $12 \%$ de las madres en el grupo de intervención $(P<0,001)$.

$\mathrm{Al}$ cabo de las 2 semanas de seguimiento solo se pudo evaluar a 103 pares de madres e hijos en el grupo testigo y a 104 pares en el grupo de intervención debido a que en el primero 14 familias habían emigrado y ocho de los niños habían fallecido, y a que en el grupo de intervención 19 familias habían emigrado y dos niños habían fallecido. En el grupo testigo, seis lactantes habían muerto de diarrea y dos de neumonía. En el grupo de intervención, la madre de un lactante había reiniciado la lactancia materna parcial en el hogar; al otro niño se le encontró muerto en la cuna durante el segundo día de su hospitalización. Quince lactantes del grupo testigo sufrieron un nuevo episodio de diarrea en el lapso de 2 semanas, en comparación con solo cuatro en el grupo que recibió lactancia materna exclusiva $(P=0,05$; razón de posibilidades $(R P)=2,92$; IC95\%: 0,86 a 10,92). 
CUADRO 2. Características de los progenitores de los lactantes que participaron en el estudio

\begin{tabular}{|c|c|c|}
\hline & $\begin{array}{l}\text { No. en } \\
\text { el grupo testigo } \\
(n=125)\end{array}$ & $\begin{array}{l}\text { No. en } \\
\text { el grupo de la intervención } \\
(n=125)\end{array}$ \\
\hline \multicolumn{3}{|l|}{ Madres } \\
\hline Edad (años) & $23,0 \pm 4,2^{a}$ & $22,1 \pm 4,0^{a}$ \\
\hline Peso $(\mathrm{kg})$ & $45,7 \pm 7,3$ & $43,8 \pm 7,4$ \\
\hline $\mathrm{IMC}^{\mathrm{b}}$ & $20,2 \pm 3,0$ & $19,5 \pm 2,9$ \\
\hline Escolaridad (años) & $4,2 \pm 4,4$ & $4,2 \pm 4,3$ \\
\hline Perímetro de la parte media del brazo $(\mathrm{cm})$ & $23,7 \pm 2,5$ & $23,0 \pm 2,5$ \\
\hline \multicolumn{3}{|l|}{ Paridad: } \\
\hline 1 & $44(35)^{c}$ & $65(52)^{d}$ \\
\hline 2 & $45(36)$ & $30(24)^{e}$ \\
\hline 3 & $20(16)$ & $22(18)$ \\
\hline$\geq 4$ & $16(12)$ & $8(6)$ \\
\hline \multicolumn{3}{|l|}{ Padres } \\
\hline Escolaridad (años) & $7,2 \pm 4,9$ & $6,9 \pm 4,9$ \\
\hline Ingresos (en takas $\left.{ }^{f}\right)$ : & $4350 \pm 5218$ & $5052 \pm 8843$ \\
\hline$\leq 3000$ & $74(59)$ & $69(56)$ \\
\hline $3001-5000$ & $26(21)$ & $30(25)$ \\
\hline$>5000$ & $25(20)$ & $23(19)$ \\
\hline \multicolumn{3}{|l|}{ Ocupación: } \\
\hline Servicios & $31(25)$ & $32(26)$ \\
\hline Negocios & $35(28)$ & $36(29)$ \\
\hline Otrag & $55(44)$ & $53(42)$ \\
\hline Sin empleo & $4(3)$ & $4(3)$ \\
\hline $\begin{array}{l}\text { a Promedio } \pm \text { desviación estándar. } \\
\text { b Índice de masa corporal. } \\
\text { ' } \text { Las cifras entre paréntesis son porcentajes. } \\
\text { d } P=0,007 \text {. } \\
\text { e } P=0,003 \text { (prueba de Mantel-Haenszel). } \\
\text { f } 40 \text { takas = US } \$ 1,00 \text {. } \\
{ }^{9} \text { Chofer de carruaje público, obrero, vendedor de tieno }\end{array}$ & Indor ambulanto & áneos. \\
\hline
\end{tabular}

CUADRO 3. Antecedentes alimentarios de los lactantes que participaron en el estudio

\begin{tabular}{lcc}
\hline & $\begin{array}{c}\text { No. en } \\
\text { el grupo testigo } \\
(n=125)\end{array}$ & $\begin{array}{c}\text { No. en } \\
\text { el grupo de la intervención } \\
(n=125)\end{array}$ \\
\hline $\begin{array}{l}\text { Recibieron calostro: } \\
\text { Sí }\end{array}$ & $116(93)^{\mathrm{a}}$ & $117(94)$ \\
No & $9(7)$ & $8(6)$ \\
Alimentos prelácteos: & $106(85)$ & $100(80)$ \\
Sí & $19(15)$ & $24(19)$ \\
No & & $17,6 \pm 18,2$ \\
Comenzaron a ingerir otra leche: & $16,5 \pm 16,6$ & $1-71$ \\
A la edad de (días) & $1-65$ & $87(70)$ \\
Recorrido (días) & & $4(3)$ \\
Razón por la cual se dieron otros alimentos: & $98(78)$ & $34(27)$ \\
No había suficiente leche materna & $4(3)$ & \\
La madre estaba enferma & $23(18)$ & $107(87)$ \\
Otrac & & $16(13)$ \\
Modo de administración: & $93(76)$ & - \\
Biberón & $26(21)$ & $2(1)$ \\
Taza y cuchara & & \\
Ambos &
\end{tabular}

${ }^{a}$ Las cifras entre paréntesis son porcentajes.

${ }^{b}$ Promedio \pm desviación estándar.

cAfecciones del pezón, bebé enfermo, diarrea, etc.

\section{DISCUSIÓN}

Este estudio se basó en la hipótesis de que la mayoría de las madres quieren amamantar a sus hijos al pecho exclusivamente durante tanto tiempo como puedan. Para ello necesitan, sin embargo, información correcta, además de la ayuda, estímulo y apoyo de la familia y de los profesionales de la salud. Por consiguiente, se ofreció orientación estructurada en materia de lactancia materna para asesorar a las madres de lactantes atendidos en un centro de tratamiento de enfermedades diarreicas, y se evaluó el efecto de la orientación. Según los resultados, el curso de orientación sobre lactancia materna dio muy buenos resultados en lo respectivo a la capacitación de las consejeras en lactancia materna, que a su vez pudieron ayudar a las madres a alcanzar la lactancia materna exclusiva mientras permanecían en el hospital.

Los hospitales de maternidad que dicen velar por el bienestar de los bebés reconocen que el estímulo y apoyo constantes son esenciales para que las madres puedan amamantar bien a sus hijos (13); deben, asimismo, tener programas para seguir dando apoyo ulterior a las madres (15). Según informes procedentes de hospitales en la India y Pakistán $(16,17)$, a la mayoría de las madres que fueron remitidas a clínicas para el manejo de la lactancia se les pudo ayudar a superar las dificultades propias del amamantamiento y a continuar la lactancia materna exclusiva. En otros países, los servicios de apoyo y orientación también han contribuido a aumentar la frecuencia de la lactancia materna exclusiva (18). Nuestro estudio difiere de los antedichos porque los niños no habían nacido en el hospital ni fueron remitidos a otros servicios para el manejo de la lactancia materna. Los investigadores aprovecharon la oportunidad de aconsejar a las madres de lactantes amamantados al pecho parcialmente que modificaran sus prácticas de amamantamiento mientras sus bebés estaban en el hospital para ser tratados por diarrea.

Antes de iniciar el estudio, pensábamos que las madres primerizas se mostrarían más receptivas al asesoramiento 
FIGURA 1. Efectos del asesoramiento sobre la alimentación del lactante (en el grupo de intervención y en el grupo testigo) al egreso y 2 semanas más tarde

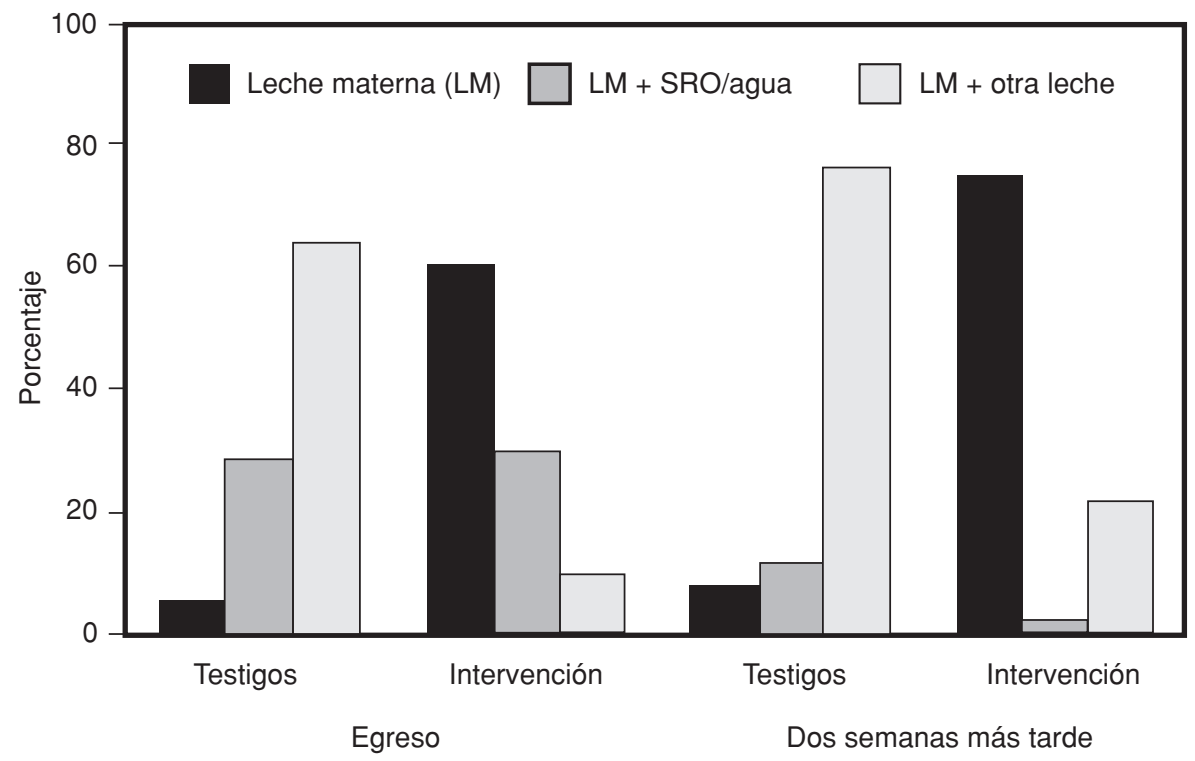

en materia de lactancia. Sin embargo, esa diferencia no se detectó cuando se compararon el orden de nacimiento y el logro de la lactancia materna exclusiva en ambos grupos, lo cual indica que la orientación en sí fue el factor decisivo en cuanto a la modificación de las prácticas de amamantamiento.

Se podría argumentar que fue más fácil modificar las prácticas de lactancia de las madres dentro del hospital porque podían dedicarse por completo a sus bebés y porque era posible controlar en cierta medida el suministro de otros tipos de leche. En el entorno doméstico, las madres tienen muchas tareas que quizá les impidan continuar practicando lo aprendido. Los resultados del seguimiento muestran, sin embargo, que en la mayoría de los casos las madres pudieron continuar con la lactancia materna exclusiva en el hogar. Se produjo otro resultado importante e interesante: aunque en el transcurso de la hospitalización todas las madres administraron a sus hijos SRO y fórmulas para lactantes, con cuchara y taza, cuando fue necesario, la mitad de las madres del grupo testigo volvieron a usar el biberón en el hogar, en comparación con un pequeño número de madres en el grupo de intervención. El hecho de que solo cuatro de los lactantes que recibieron el pecho exclusivamente sufrieran otro episodio de diarrea en las 2 semanas posteriores a su egreso pone de manifiesto el efecto protector de la lactancia materna exclusiva $(2,5)$, aun dentro de un período de seguimiento breve.

Por razones obvias, a las personas encargadas de evaluar el estudio no se les pudo ocultar la asignación a los grupos. Fue necesario mantener a los pacientes de ambos grupos en partes separadas del hospital para evitar la Las instrucciones médicas y las hojas de consumo alimentario también proporcionaron información sobre la dieta de los lactantes. Cualquier sesgo que pudiera existir sería, sin embargo, sumamente pequeño, ya que los evaluadores mismos no participaron ni en el asesoramiento que se ofreció durante la hospitalización ni en la primera visita de seguimiento.

Puede que existan dudas en torno a la reproducibilidad del programa, ya que es poco factible que en la mayor parte de los hospitales las consejeras en lactancia materna puedan hacer visitas a domicilio de forma sistemática. No obstante, la visita de seguimiento fue contaminación por el mensaje ajeno. valiosa; las consejeras consideraron que el refuerzo de los mensajes transmitidos durante el asesoramiento y la sensación de seguridad que despertaban contribuyeron a incrementar la confianza de las madres y les permitieron continuar con la lactancia materna exclusiva. De no haberse hecho esa visita, es posible que no se hubiera obtenido un éxito tan grande.

La falta de confidencialidad durante las sesiones de asesoramiento en el hospital puede haber sido un factor limitante. Es posible que esto suceda también en otros países en desarrollo donde por tradición las mujeres no pueden hablar libremente en presencia de extraños. Puede que dos o tres sesiones breves en tales circunstancias no siempre basten para determinar qué causa subyacente lleva a las madres a creer que "no tienen suficiente leche", factor que probablemente explique el fracaso en algunos casos.

En conclusión, se puede decir que la intervención en sí fue muy eficaz y que, por lo tanto, se debe incorporar a los programas de salud maternoinfantil, ya que reduce notablemente la morbilidad y mortalidad de los lactantes. El personal de salud de las unidades de atención pediátrica y de enfermedades diarreicas debe recibir capacitación sobre lactancia materna y asesorar a las madres para ayudarlas a mejorar las prácticas de alimentación de los lactantes en alto riesgo. Es preciso explorar la posibilidad de enseñar a un grupo selecto de madres a servir de asesoras en la comunidad, puesto que sería una manera más económica y práctica de ayudar y estimular a las madres a iniciar la lactancia materna exclusiva inmediatamente después del parto. También podrían ser fuente de apoyo ulterior para las madres que reciben asesoramiento en materia de lactancia materna en el hospital (como lo hicieron en el presente estudio) y podrían, por ese medio, contribuir a prevenir las enfermedades diarreicas en niños lactantes.

Agradecimiento. La investigación fue patrocinada por la OMS (subvenciones No. GL/RES/CDD/317/RB/ 
94.B/300 y GL/RES/CDD/317/VC/ 94.B/300) y el Centro Internacional para la Investigación de las Enfermedades Diarreicas, Bangladesh. Este Centro recibe el apoyo financiero de organismos de cooperación de los gobiernos de Alemania, Arabia Saudita, Australia, Bangladesh, Bélgica, Canadá, China, Estados Unidos de América, Japón, Noruega, Países Bajos, Reino Unido, República de Corea, Suecia y Suiza; de organismos internacionales como el Banco Asiático de Desarrollo, el Fondo de Población de las Huffman SL, Martin LH. First feedings: opti-
mal feeding of infants and toddlers. Nutr Res 1994:14:127-159.

2. Feachem RG, Koblinsky MA. Interventions for the control of diarrhoeal diseases among young children: promotion of breast-feeding. Bull World Health Organ 1984;62:271-291.

3. Ketsela $T$, et al. Patterns of breast feeding in western Ethiopia and their relationship to acute diarrhoea in infants. J Trop Pediatr 1990; 36:180-183.

4. Mahmood DA, et al. Infant feeding and risk of severe diarrhoea in Basrah city, Iraq: a casecontrol study. Bull World Health Organ 1989; 67:701-706.

5. Victora GC, et al. Evidence for protection by breast-feeding against infant deaths from infectious diseases in Brazil. Lancet 1987;2: 319-322.

6. Clemens JD, et al. Breast-feeding as a determinant of severity in shigellosis: evidence for protection throughout the first three years of
Naciones Unidas (FNUAP), el Fondo del Golfo Árabe, la OMS, el Organismo Internacional de Energía Atómica, el Programa de las Naciones Unidas para el Desarrollo (PNUD), la Unión Europea y el Fondo de las Naciones Unidas para la Infancia (UNICEF); de fundaciones privadas, como la Child Health Foundation, el Consejo de Población, la Fundación Ford, la Fundación Rockefeller y la Fundación Sasakawa; y de organizaciones privadas como el American Express Bank, Bayer AG, CARE, la Cruz Roja Suiza, Family

\section{REFERENCIAS}

life in Bangladeshi children. Am J Epidemiol 1986;123:710-720.

7. Clemens JD, et al. Breast-feeding and the risk of severe cholera in rural Bangladeshi children. Am I Epidemiol 1990;131: 400-411.

8. de Zoysa I, et al. Why promote breastfeeding in diarrhoeal disease control programmes? Health Policy Plann 1991;6:371-379.

9. Brown $\mathrm{KH}$, et al. Infant-feeding practices and their relationship with diarrheal and other diseases in Huascar (Lima), Peru. Pediatrics 1989;83:31-40.

10. Talukder MQ-K. Bangladesh campaign for protection and promotion of breastfeeding. Bangladesh J Child Health 1992;16:25-31.

11. Feachem RG, et al. Diarrhoeal disease control: reviews of potential interventions. Bull World Health Organ 1984;637-640.

12. Feachem RG. Preventing diarrhoea: what are the policy options? Health Policy Plann 1986; 1:109-117.
Health International, Helen Keller International, Procter Gamble Rand Corporation, Sandoz, la Universidad de California en Davis y la Universidad Johns Hopkins.

Agradecemos a la doctora Felicity Savage y al doctor José Martines de la OMS sus aportes y sugerencias; a Tanzila Faruque y Shahara Bano su excelente trabajo de orientación sobre lactancia; a los doctores S. Laston y G. Fuchs la revisión de este trabajo, y a $\mathrm{M}$. A. R. Patwary su colaboración en las funciones de secretariado.
13. United Nations Children's Fund. Take the baby-friendly initiative! New York: UNICEF; 1992.

14. Labbok M, Krasovec K. Towards consistency in breastfeeding definitions. Studies Fam Plann 1990;21:226-230

15. World Health Organization, United Nations Children's Fund. Protecting, promoting and supporting breast-feeding: the special role of maternity services: a Joint WHO/UNICEF Statement. Geneva: WHO; 1989. clinic: positive reinforcement to hospital breastfeeding practices. Indian Pediatr 1994;31: 1385-1389.

17. Abbas KA. Counselling in a hospital setting. Dialogue Diarrhoea 1994-95;59:3.

18. Valdes V, et al. The impact of a hospital and clinic-based breastfeeding promotion programme in a middle-class urban environment. J Trop Pediatr 1993;39:142-151.
16. Nanavati RN, et al. Lactation management

ABSTRACT Lactation counsellors were trained to advise mothers of partially breast-fed infants who were admitted to hospital because of diarrhoea, so that they could start exclusive breast-feeding during their hospital stay. Infants $(n=250)$ up to 12 weeks of age were randomized to intervention and control groups. Mothers in the intervention group were individually advised by the counsellors while mothers in the control group received only routine group health education. During follow-up at home by the counsellors a week later, only the mothers in the intervention group were counselled. All the mothers were evaluated for infant feeding practices at home two weeks after discharge.

Among the 125 mother-infant pairs in each group, $60 \%$ of mothers in the intervention group were breast-feeding exclusively at discharge compared with only $6 \%$ in the control group $(P<0.001)$; two weeks later, these rates rose to $75 \%$ and $8 \%$ in the intervention and control groups, respectively $(P<0.001)$. However, $49 \%$ of mothers in the control group reverted back to bottle-feeding compared with $12 \%$ in the intervention group $(P<0.001)$. Thus, individual counselling had a positive impact on mothers to start exclusive breast-feeding during hospitalization and to continue the practice at home.

Maternal and child health facilities should include lactation counselling as an integral part of their programmes to improve infant feeding practices. 